\title{
REMOVABLE SINGULARITIES IN THE NEVANLINNA CLASS AND IN THE HARDY CLASSES
}

\author{
JUHANI RIIHENTAUS
}

(Communicated by Irwin Kra)

\begin{abstract}
We show that certain sets in $\mathbf{C}^{n}, n \geq 2$, which we call $n$-small, are removable singularities for holomorphic functions in the Nevanlinna class. Since our class of sets includes polar sets (in $\mathbf{R}^{2 n}$ ) our result includes the previous removable singularity results for the Nevanlinna class. We give also a related result for a subclass of the Hardy class.
\end{abstract}

1. Throughout this paper, $G$ is an open set in $\mathbf{C}^{n}, n \geq 1$, and $E$ is closed in G. Parreau [12, Théorème 20, p. 182] gave essentially the following result: If $n=1, E$ is polar, and $f$ is a holomorphic function in $G \backslash E$ such that $\log ^{+}|f|$ has a harmonic majorant in $G \backslash E$, then $f$ has a meromorphic extension to $G$. In answering a question of Cima and Graham [2, Remarks 7.4, p. 255], Parreau's theorem was in $[7$, Theorem 3.4, p. 477] extended to the case $n \geq 2$ and $E$ polar in $\mathbf{R}^{2 n}$.

The purpose of this note is to give a result, Theorem 1 below, which contains the above result as a special case. In Theorem 1 the exceptional set $E$ is allowed to be slightly larger, that is $n$-small (for the definition see [14, Definition $2.2, \mathrm{p}$. 101] or $\S 3$ below). However, we must then replace the condition that $\log ^{+}|f|$ has a harmonic majorant in $G \backslash E$ by the condition that the (Riesz) measure $\Delta \log ^{+}|f|$ has locally finite mass near the exceptional set $E$. In addition, we give in $\S 6$ a slight generalization to [8, Corollary 3.6, p. 301].

2. Let $u$ be a subharmonic function in $G \backslash E$. One says that the (Riesz) measure $\Delta u$ has locally finite mass near $E$, if $\Delta u(D \backslash E)<\infty$ for each open set $D \Subset G$ (relatively compact in $G$ ). From [1, p. 283] (see also [7, Lemma 3.3, p. 476]), it follows that if $\Delta u$ has locally finite mass near $E$, then $u$ has locally a harmonic majorant near $E$ (that is, for each open set $D \Subset G$ there is a harmonic function $h$ in $D \backslash E$ such that $u \leq h$ in $D \backslash E)$. The converse holds in the important case when $E$ is polar in $\mathbf{R}^{2 n}$ (see [1, p. 283] or the proof of Corollary 1 below), but not in general. To get a simple example, set $E^{\prime}=\{z=x+i y \in \mathbf{C}|| x|=| y \mid\}, u(z)=$ $-\log (\max \{|x|,|y|\})$ and $h(z)=\log \sqrt{2}-\log |z|$. Then in $\mathbf{C} \backslash E^{\prime}$ the subharmonic function $u$ has the harmonic majorant $h$. However, one sees easily that $\Delta u$ does not have locally finite mass near $E^{\prime}$.

Received by the editors September 17, 1984 and, in revised form, December 16, 1986.

1980 Mathematics Subject Classification (1985 Revision). Primary 32D20.

Key words and phrases. Holomorphic function, meromorphic function, removable singularity set, polar set, Hausdorff measure, Riesz mass, harmonic majorant. 
3. For $F \subset \mathbf{C}$, set $C^{1}(F)=\operatorname{cap}^{*} F$, where cap* is the outer logarithmic capacity (for the definition of cap*, see [5, pp. 210, 273]). If $F \subset \mathbf{C}^{n}, n \geq 2$, then set

$$
C^{n}(F)=\max _{1 \leq j \leq n} H_{2}\left\{z_{j} \in \mathbf{C} \mid C^{n-1}\left\{Z_{j} \in \mathbf{C}^{n-1} \mid\left(z_{j}, Z_{j}\right) \in F\right\}>0\right\} .
$$

Here, and in the sequel, $z=\left(z_{1}, \ldots, z_{j}, \ldots, z_{n}\right)=\left(z_{j}, Z_{j}\right), 1 \leq j \leq n$, and $H_{\alpha}$ is the $\alpha$-dimensional Hausdorff outer measure. We say that $F \subset \mathbf{C}^{n}$ is $n$-small, if $C^{n}(F)=0$.

Lemma 1 [7, Proposition 2.3, p. 472]. An $\boldsymbol{F}_{\sigma}$-set $F \subset \mathbf{C}^{n}, n \geq 2$, is $n$-small if and only if for each $j, 1 \leq j \leq n, H_{2 n-2}\left(F_{j}\right)=0$, where

$$
F_{j}=\left\{Z_{j} \in \mathbf{C}^{n-1} \mid \operatorname{cap}^{*}\left\{z_{j} \in \mathbf{C} \mid\left(z_{j}, Z_{j}\right) \in F\right\}>0\right\} .
$$

With the aid of this lemma it follows from a result of Mattila [10, Corollary 3.3, p. 263] (see also [15, Lemma 6, p. 115]) that polar sets in $\mathbf{R}^{2 n}$ are $n$-small.

If the Hausdorff measure $\mathrm{H}_{2}$ in (A) is replaced by the outer logarithmic capacity cap*, a set function which is sometimes denoted by $g_{n}$ is obtained. Sets $E$ for which $g_{n}(E)=0$ have been used as exceptional sets at least in $[1,16,17$, and 14]. If $F \subset \mathbf{C}^{n}$ and $g_{n}(F)=0$, then clearly $F$ is $n$-small. On the other hand, there are sets $F \subset \mathbf{C}^{n}$ with $g_{n}(F)=0$ which are not even polar. In fact, with the help of [3, Theorem 2, p. 118] one can construct a compact set $F \subset \mathbf{C}^{2}$ of Hausdorff dimension 4 such that $g_{2}(F)=0$ and such that for each $z \in \mathbf{C}$ the sections $F \cap(\mathbf{C} \times\{z\})$ and $F \cap(\{z\} \times \mathbf{C})$ contain at most one point.

4. Next we give the lemmas we need in the sequel.

LEMMA 2 [18, THEOREM 1.2, p. 16]. Let $G^{\prime}$ be a domain of $\mathrm{C}^{n-1}, n \geq 2$, and $F \subset G^{\prime}$. Suppose $j \in \mathbf{N}, 1 \leq j \leq n, r_{j}^{\prime}, r_{j}^{\prime \prime} \in \mathbf{R}, 0<r_{j}^{\prime}<r_{j}^{\prime \prime}$, and $z_{j}^{0} \in \mathbf{C}$. Let $f$ be a holomorphic function in the open set

$$
V\left(G^{\prime}, z_{j}^{0}, r_{j}^{\prime}, r_{j}^{\prime \prime}\right)=\left\{z=\left(z_{j}, Z_{j}\right) \in \mathbf{C}^{n} \mid z_{j} \in A\left(z_{j}^{0}, r_{j}^{\prime}, r_{j}^{\prime \prime}\right), Z_{j} \in G^{\prime}\right\}
$$

such that for each $Z_{j} \in F$ the holomorphic function $f_{Z_{j}}: A\left(z_{j}^{0}, r_{j}^{\prime}, r_{j}^{\prime \prime}\right) \rightarrow \mathbf{C}$,

$$
f_{Z_{j}}\left(z_{j}\right)=f\left(z_{j}, Z_{j}\right)=f(z)
$$

has a meromorphic extension to $B^{2}\left(z_{j}^{0}, r_{j}^{\prime \prime}\right)$. If $F$ is not contained in a countable union of analytic subvarieties in $G^{\prime}$ of codimension $\geq 1$, then $f$ has a meromorphic extension to the open set

$$
V\left(G^{\prime}, z_{j}^{0}, r_{j}^{\prime \prime}\right)=\left\{z=\left(z_{j}, Z_{j}\right) \in \mathbf{C}^{n} \mid z_{j} \in B^{2}\left(z_{j}^{0}, r_{j}^{\prime \prime}\right), Z_{j} \in G^{\prime}\right\} .
$$

Here, $B^{2}\left(z_{0}, r\right)$ is the disc in $\mathbf{C}$ with center $z_{0}$ and radius $r$, and $A\left(z_{0}, r_{1}, r_{2}\right)$ is the annulus $B^{2}\left(z_{0}, r_{2}\right) \backslash \bar{B}^{2}\left(z_{0}, r_{1}\right)$. Following Siu [18, p. 17] we define the radius of meromorphy as follows (note that also other definitions are used, see for example $\left[4\right.$, p. 578]). Let $f$ be a holomorphic function in the set $V\left(G^{\prime}, z_{j}^{0}, r_{j}\right)$ where $G^{\prime}, j$, and $z_{j}^{0}$ are as above, and $r_{j} \in \mathbf{R}_{+}$. For each $Z_{j} \in G^{\prime}$ the radius of meromorphy $\rho_{j}\left(Z_{j}\right)$ is the supremum of all $\rho>0$ such that $f$ has a meromorphic extension to a neighborhood of the set

$$
V\left(Z_{j}, z_{j}^{0}, \rho\right)=\left\{z=\left(z_{j}, Z_{j}\right) \in \mathbf{C}^{n} \mid z_{j} \in B^{2}\left(z_{j}^{0}, \rho\right)\right\} .
$$


With this notation, we have

LEMMA 3 [18, PROPOSITION 1.4 AND REMARK 1.5, pp. 17-18]. The function $v_{j}: G^{\prime} \rightarrow[-\infty, \infty), v_{j}\left(Z_{j}\right)=-\log \rho_{j}\left(Z_{j}\right)$, is subharmonic.

5. If $f$ is a holomorphic function in $G \backslash E$ and has a meromorphic extension $f^{*}$ to $G$, then the measure $\Delta \log ^{+}|f|$ has locally finite mass near $E$. This follows from the fact that each point $z_{0} \in G$ has a neighborhood $U$ in $G$ such that $\log ^{+}\left|f^{*}\right|$ has a pluriharmonic majorant in $U \backslash N\left(f^{*}\right)$. Here, $N\left(f^{*}\right)$ is the nonsmooth set of $f^{*}$; see, for example, [19, pp. 184-185]. As for the converse, we have

THEOREM 1. Let $E$ be n-small. Let $f$ be a holomorphic function in $G \backslash E$. If $\Delta \log ^{+}|f|$ has locally finite mass near $E$, then $f$ has a meromorphic extension to $G$.

ProOF. If $n=1$, then $E$ is polar. Thus $\log ^{+}|f|$ has locally a harmonic majorant near $E$, and the theorem follows from [12, Théorème 20, p. 182].

Suppose then $n \geq 2$. It is sufficient to show that each point $z^{*} \in E$ has a neighborhood $V$ such that $f \mid V \backslash E$ has a meromorphic extension to $V$. As for the notation not explained in the sequel, see above or [7].

Take $z^{*} \in E$ arbitrarily and choose $r=\left(r_{1}, \ldots, r_{n}\right) \in \mathbf{R}_{+}^{n}$ such that

$$
U=D^{n}\left(z^{*}, 2 r\right)=B^{2}\left(z_{1}^{*}, 2 r_{1}\right) \times \cdots \times B^{2}\left(z_{n}^{*}, 2 r_{n}\right) \Subset G .
$$

For shortness, we write $u=\log ^{+}|f| \mid U \backslash E$. Since $\Delta u(U \backslash E)<\infty$, one sees, proceeding as Cegrell [1, proof of Theorem, pp. 284-285], using a nondecreasing sequence of nonnegative testfunctions in $U \backslash E$ tending to 1 , using the $n$-subharmonicity of $u$, Fubini's theorem, and the Monotone convergence theorem, that for each $j, 1 \leq j \leq n$, there is a set $B_{j} \subset \mathbf{C}^{n-1}$ such that $H_{2 n-2}\left(B_{j}\right)=0$ and that for each $Z_{j} \in U\left(z_{j}^{*}\right) \backslash B_{j}$ the measure $\Delta u_{Z_{j}}$ has locally finite mass near the section $(E \cap U)\left(Z_{j}\right)$. For a detailed discussion of this, see [7, proof of Theorem 3.4, pp. 477-478]. Since $E$ is $n$-small, by Lemma 1 we may suppose that the section $(E \cap U)\left(Z_{j}\right)$ is polar in $\mathbf{C}$ for each $Z_{j} \in U\left(z_{j}^{*}\right) \backslash B_{j}$. Above we have used the notation

$$
\begin{aligned}
& U\left(z_{j}^{*}\right)=\left\{Z_{j} \in \mathbf{C}^{n-1} \mid z=\left(z_{j}^{*}, Z_{j}\right) \in U\right\} \\
& (E \cap U)\left(Z_{j}\right)=\left\{z_{j} \in \mathbf{C} \mid z=\left(z_{j}, Z_{j}\right) \in U \cap E\right\}
\end{aligned}
$$

for the sections of $U$ in $\mathbf{C}^{n-1}$ and of $E \cap U$ in $\mathbf{C}$, respectively. Take $Z_{j}^{\prime} \in U\left(z_{j}^{*}\right) \backslash B_{j}$ arbitrarily. Since the section $(E \cap U)\left(Z_{j}^{\prime}\right)$ is then polar in $\mathbf{C}$ and $E$ is closed in $G$, there are $r_{j}^{\prime}, r_{j}^{\prime \prime}, 0<r_{j}^{\prime}<r_{j}^{\prime \prime}<2 r_{j}$ and a (connected) neighborhood $W \subset U\left(z_{j}^{*}\right)$ of $Z_{j}^{\prime}$ such that $V\left(W, z_{j}^{*}, r_{j}^{\prime}, r_{j}^{\prime \prime}\right) \Subset G \backslash E$. Since for each $Z_{j} \in W \backslash B_{j}$ the measure $\Delta u_{Z_{j}}$ has locally finite mass near the polar set $(E \cap U)\left(Z_{j}\right)$, as shown above, we see as in the case $n=1$ treated above, that the holomorphic functions $f_{Z_{j}} \mid B^{2}\left(z_{j}, 2 r_{j}\right) \backslash(E \cap$ $U)\left(Z_{j}\right), Z_{j} \in W \backslash B_{j}$, have meromorphic extensions to $B^{2}\left(z_{j}^{*}, 2 r_{j}\right)$. Invoking this and the facts that $f$ is holomorphic in $V\left(W, z_{j}^{*}, r_{j}^{\prime}, r_{j}^{\prime \prime}\right)$ and $H_{2 n-2}\left(B_{j}\right)=0$, we see by Lemma 2 that $f$ has a meromorphic extension to the set $V\left(W, z_{j}^{*}, r_{j}^{\prime \prime}\right)$. Since $r_{j}^{\prime \prime}$ can be chosen arbitrarily near to $2 r_{j}^{\prime}$, we have shown the following: For each $j$, $1 \leq j \leq n$, and $Z_{j} \in U\left(z_{j}^{*}\right) \backslash B_{j}$, the function $f$ has a meromorphic extension to a neighborhood of the set $V\left(Z_{j}, z_{j}^{*}, 2 r_{j}\right)$. We refer to this result as condition (B). Take $z_{0} \in D^{n}\left(z^{*}, r\right)$ and $r^{0}=\left(r_{1}^{0}, \ldots, r_{n}^{0}\right) \in \mathbf{R}_{+}^{n}$ such that $V_{0}=D^{n}\left(z_{0}, r^{0}\right) \Subset$ $D^{n}\left(z^{*}, r\right) \backslash E$. By (B) we see that $\rho_{1}\left(Z_{1}\right) \geq r_{1}$ for each $Z_{1} \in V_{0}\left(z_{1}^{0}\right) \backslash B_{1}$. Thus 
by Lemma 3 and, for example, by [6, Proposition $\left.2 b^{\prime}\right)$, p. 10], $\rho_{1}\left(Z_{1}\right) \geq r_{1}$ for all $Z_{1} \in V_{0}\left(z_{1}^{0}\right)$, the section of $V_{0}$. Thus $f$ has a meromorphic extension $f_{1}$ to $V_{1}=D^{n}\left(z_{0}, r^{1}\right)$, where $r^{1}=\left(r_{1}, r_{2}^{0}, \ldots, r_{n}^{0}\right)$. Observe that $z_{1}^{*} \in B^{2}\left(z_{1}^{0}, r_{1}\right)$. For the induction step suppose that $1 \leq k<n$ and $r^{k}=\left(r_{1}, \ldots, r_{k}, r_{k+1}^{0}, \ldots, r_{n}^{0}\right) \in$ $\mathbf{R}_{+}^{n}$, and $f_{k}$ is a holomorphic extension of $f$ to $V_{k}=D^{n}\left(z_{0}, r^{k}\right)$. Observe that $z_{j}^{*} \in B^{2}\left(z_{j}^{0}, r_{j}\right), j=1, \ldots, k$. As above, using (B) we see that $\rho_{k+1}\left(Z_{k+1}\right) \geq r_{k+1}$ for all $Z_{k+1} \in V_{k}\left(z_{k+1}^{0}\right)$. Thus $f_{k}$ and hence also $f$ has a meromorphic extension $f_{k+1}$ to $V_{k+1}=D^{n}\left(z_{0}, r^{k+1}\right)$, where $r^{k+1}=\left(r_{1}, \ldots, r_{k+1}, r_{k+2}^{0}, \ldots, r_{n}^{0}\right)$. Moreover, $z_{j}^{*} \in B^{2}\left(z_{j}^{0}, r_{j}\right), j=1, \ldots, k+1$, and $V_{k+1} \subset U$. Thus the induction step is complete. Since $V_{n}=D^{n}\left(z_{0}, r^{n}\right)$ is a neighborhood of $z^{*}$, the proof is finished.

REMARK. The proof can also be based on [4, Theorem 2.9, p. 578] instead of Lemmas 2 and 3.

COROLlARY 1 [7, THEOREM 3.4, p. 477]. Let $E$ be polar (respectively $n$ small). Let $f$ be a holomorphic function in $G \backslash E$. If $\log ^{+}|f|$ has a harmonic majorant (respectively $n$-superharmonic majorant which is $\not \equiv \infty$ on each component of $G \backslash E)$, then $f$ has a meromorphic extension to $G$.

ProOF. By [6, Theorem 2, p. 25] (respectively [14, Theorem 4.1, p. 105]) the harmonic (respectively $n$-superharmonic) majorant $h$ to $\log ^{+}|f|$ has a superharmonic extension $h^{*}$ to $G$. Similarly, the subharmonic (respectively $n$-subharmonic) function $\log ^{+}|f|-h$ has a subharmonic extension $h_{1}^{*}$ to $G$. Since $\log ^{+}|f|=h^{*}+h_{1}^{*}$ in $G \backslash E$ and $-\Delta h^{*}$ and $\Delta h_{1}^{*}$ are measures in $G$, the corollary follows.

6. Computing the Laplacian, using Kametani's extension result [11, Theorem 2, p. 10, and Remark, p. 11] and proceeding as in the proof of Theorem 1 above, the following result will be proved in [8, Corollary 3.6, p. 301]: Let $H_{2 n-1}(E)=0$. Let $f$ be a holomorphic function in $G \backslash E$. If for some $p>0$ the measure $\Delta|f|^{p}$ has locally finite mass near $E$, then $f$ has a holomorphic extension to $G$. The proof works also for measures $\Delta\left(\log ^{+}|f|\right)^{p}, p>1$ (but not if $p=1$ ), instead of the measures $\Delta|f|^{p}, p>0$. In fact, one can easily give a unified result which contains both cases:

THEOREM 2 (WITH J. HYVÖNEN). Let $H_{2 n-1}(E)=0$. Let $f$ be a holomorphic function in $G \backslash E$. Let $\varphi:[-\infty, \infty) \rightarrow \mathbf{R}$ be a nondecreasing function such that $\varphi \mid \mathbf{R}$ is strongly convex. Suppose that $\varphi \mid(\rho, \infty)$ is twice continuously differentiable for some $\rho \in \mathbf{R}$. If the measure $\Delta(\varphi \circ \log |f|)$ has locally finite mass near $E$, then $f$ has a holomorphic extension to $G$.

OUTLINE OF PROOF. In view of the proof of Theorem 1 one may restrict to the case $n=1$ (instead of the radius of meromorphy one can as well consider the radius of holomorphy). Take an open set $D \Subset G$ and set $U=\{z \in D \backslash E|\log | f(z) \mid>\rho\}$, where $\rho \in \mathbf{R}$ is as in the theorem. Using a well-known inequality, computing the Laplacian and using the fact that $\Delta(\varphi \circ \log |f|)(U) \leq \Delta(\varphi \circ \log |f|)(D \backslash E)<\infty$, one gets

$$
\begin{aligned}
\int_{f(U)} \varphi^{\prime \prime}(\log |w|) \frac{1}{|w|^{2}} d m_{2}(w) & \leq \int_{U} \varphi^{\prime \prime}(\log |f(z)|) \frac{\left|f^{\prime}(z)\right|^{2}}{|f(z)|^{2}} d m_{2}(z) \\
& =\Delta(\varphi \circ \log |f|)(U)<\infty
\end{aligned}
$$


On the other hand, since $\varphi$ is strongly convex,

$$
\int_{\mathbf{C} \backslash B^{2}\left(0, r_{0}\right)} \varphi^{\prime \prime}(\log |w|) \frac{1}{|w|^{2}} d m_{2}(w)=2 \pi \int_{r_{0}}^{\infty} \varphi^{\prime \prime}(\log r) \frac{1}{r} d r=\infty
$$

for each $r_{0}>e^{\rho}$. Thus $f$ omits a set of positive measure and has by the cited result of Kametani a meromorphic extension to $D$. Since this extension must be holomorphic, the theorem follows.

REMARK. Proceeding as in [8] one can also consider more general exceptional sets.

We want to thank Jaakko Hyvönen for a discussion.

\section{REFERENCES}

1. U. Cegrell, Removable singularity sets for analytic functions having modulus with bounded Laplace mass, Proc. Amer. Math. Soc. 88 (1983), 283-286.

2. J. A. Cima and I. R. Graham, On the extension of holomorphic functions with growth conditions across analytic subvarieties, Michigan Math. J. 28 (1981), 241-256.

3. R. O. Davies and H. Fast, Lebesgue density influences Hausdorff measure; Large sets surface like from many directions, Mathematika 25 (1978), 116-119.

4. R. Harvey and B. Shiffman, A characterization of holomorphic chains, Ann. of Math. 99 (1974), $553-587$.

5. W. K. Hayman and P. B. Kennedy, Subharmonic functions. I, Academic Press, London, 1976.

6. M. Hervé, Analytic and plurisubharmonic functions in finite and infinite dimensional spaces, Springer-Verlag, Berlin, 1970.

7. J. Hyvönen and J. Riihentaus, On the extension in the Hardy classes and in the Nevanlinna class, Bull. Soc. Math. France 112 (1984), 469-480.

8. __ Removable singularities for holomorphic functions with locally finite Riesz mass, J. London Math. Soc. (2) 35 (1987), 296-302.

9. P. Järvi, Removable singularities for $H^{p}$-functions, Proc. Amer. Math. Soc. 86 (1982), 596-598.

10. P. Mattila, An integral inequality for capacities, Math. Scand. 53 (1983), 256-264.

11. K. Noshiro, Cluster sets, Springer-Verlag, Berlin, 1960.

12. M. Parreau, Sur les moyennes des fonctions harmoniques et analytiques et la classification des surfaces de Riemann, Ann. Inst. Fourier (Grenoble) 3 (1951), 103-197.

13. J. Riihentaus, On the extension of holomorphic functions with growth conditions, J. London Math. Soc. (2) 27 (1983), 281-288.

14. $\ldots$, On the extension of separately hyperharmonic functions and $H^{p}$-functions, Michigan Math. J. 31 (1984), 99-112.

15. A. Sadullaev, Rational approximation and pluripolar sets, Mat. Sb. (N.S.) 119 (1982), 96-118. (Russian)

16. D. Singman, Exceptional sets in a product of harmonic spaces, Math. Ann. 262 (1983), 29-43.

17. _ Removable singularities for n-harmonic functions and Hardy classes in polydiscs, Proc. Amer. Math. Soc. 90 (1984), 299-302.

18. Y.-T. Siu, Techniques of extension of analytic objects, Dekker, New York, 1974.

19. H. Whitney, Complex analytic varieties, Addison-Wesley, Reading, Mass., 1972.

Department of Mathematics, University of Joensud, SF-80101 JoensuU, FinLAND

Department of Mathematics, University of Oulu, SF-90570 Oulu, Finland 